\title{
APPLICATION OF QUEUEING SYSTEM FOR INCREASING RELIABILITYINDEX OF POWER SUPPLY SYSTEMS
}

\author{
Alexey Efanov ${ }^{1}$, Nikolay Bogatyrev ${ }^{2}$, Sergey Yastrebov ${ }^{1}$, Viktor Yarosh $^{1}$ \\ ${ }^{1}$ Stavropol State Agrarian University, Russia; \\ ${ }^{2}$ Kuban State Agrarian University named after I. T. Trubilin, Russia \\ yefanov@mail.ru, bogatyrevn@yandex.ru,yastrsergej@yandex.ru, yarviktor@yandex.ru
}

\begin{abstract}
The paper considers the use of a delay queuing system for increasing the reliability of power supply systems for agricultural electricity consumers, which has been estimated using SAIDI. At the same time, both the mode of current operation of distribution electric networks and the mode of emergence of mass failures in electric networks under the influence of unfavorable weather factors have been considered. The reliability of power supply has been estimated by the value of SAIDI, and the change in this index occurs by reducing the time to eliminate interruptions in power supply to consumers by determining the required number of repair personnel. The determination of the number of repair personnel has been carried out using a delay queuing system in transient modes. The choice of the required number of repair personnel has been based on the numerical solution of differential equations describing the delay queuing system, using the criterion of the optimal delay in servicing of requests for eliminating interruptions in power supply to consumers. The calculations have been carried out both for the stationary value of the request flow parameter for eliminating interruptions in power supply, and for the cases of step change in the request flow parameter. According to the results of the calculations, the dependence of SAIDI on the number of repair personnel and the optimal number of repair personnel at economically justified costs for eliminating interruptions in power supply to consumers have been determined.
\end{abstract}

Keywords: power supply system, power distribution network, SAIDI, reliability, queueing system.

\section{Introduction}

The development of agriculture and rural territories is impossible without reliable power supply. The reliability of power supply is influenced by many factors, such as electric distribution network schematic diagram, the lifetime of the electrical equipment of the electrical networks, the organization of the operation of the electrical equipment, weather conditions. In addition to the above, an important factor is the availability of distributed (standby) generation [1]. At the same time, in the event of various non-standard situations in the operation of rural distribution electric networks, for example, unfavorable weather conditions [2], one of the bases for ensuring a sufficient level of reliability is the timely restoration of power supply to consumers when they are cut off.

The choice of such a reliability index, according to which it is necessary to assess the reliability of consumers' power supply, is also important. The most used are indexes such as SAIFI, SAIDI, CAIDI, CAIFI [3-5] for the so-called steady faulting (which are eliminated by the operational, repair or maintenance personnel) and MAIFI, which shows the number of disconnections of consumers of short duration (no more than $1 \mathrm{~min}$ ), which are eliminated by the actions of network automation.

The aim of this paper is to determine ways to increase the reliability indexes of power supply to consumers, based on average estimates of SAIDI by determining the required number of personnel involved in the elimination of mass failures in power supply systems. In this case, it is necessary to strike a balance between the number of personnel involved and the cost of it, and the possible losses for agricultural consumers from interruptions in power supply.

\section{Materials and methods}

To assess the reliability of power supply to consumers, such an index as SAIDI is used [3]:

$$
S A I D I=\frac{\sum T_{i} N_{i}}{N_{T O T}}
$$

where $T_{i}$-duration of interruptions in power supply to consumers in the amount of $N_{i}$, located in the $i$ node;

$N_{T O T}$ - total number of consumers served by the power supply system.

In general, this index is calculated specifically for a given period of time (usually 1 year), for all types of consumer disconnections. Cutoffs can be planned and unscheduled (emergency), which, in 
turn, are divided into those arising during the current operation, and arising under unfavorable weather conditions, such as during a hurricane, glaze-clear ice, snowfall, etc. Scheduled cutoffs are carried out in order to perform scheduled preventive repairs, which, if properly organized, are accomplished on all connected equipment of the power supply system, and the period of their execution is about 1 time in 6-12 years and depends on the type of equipment used, the duration of repairs usually does not exceed 8 hours. Emergency cutoffs under normal operating conditions have a cutoff frequency (average) once in 5 years, with an average recovery time of about 4 hours. But a strong enough influencing factor on SAIDI is massive failure in unfavorable weather conditions.

In the case of technological failures (TF) caused by unfavorable weather factors, the distribution of the recovery time and the rate of failures occurred parameter can have other values, for which it is necessary to analyze the data of enterprises using electric networks. The results of the data analysis are shown in Figure 1[6].

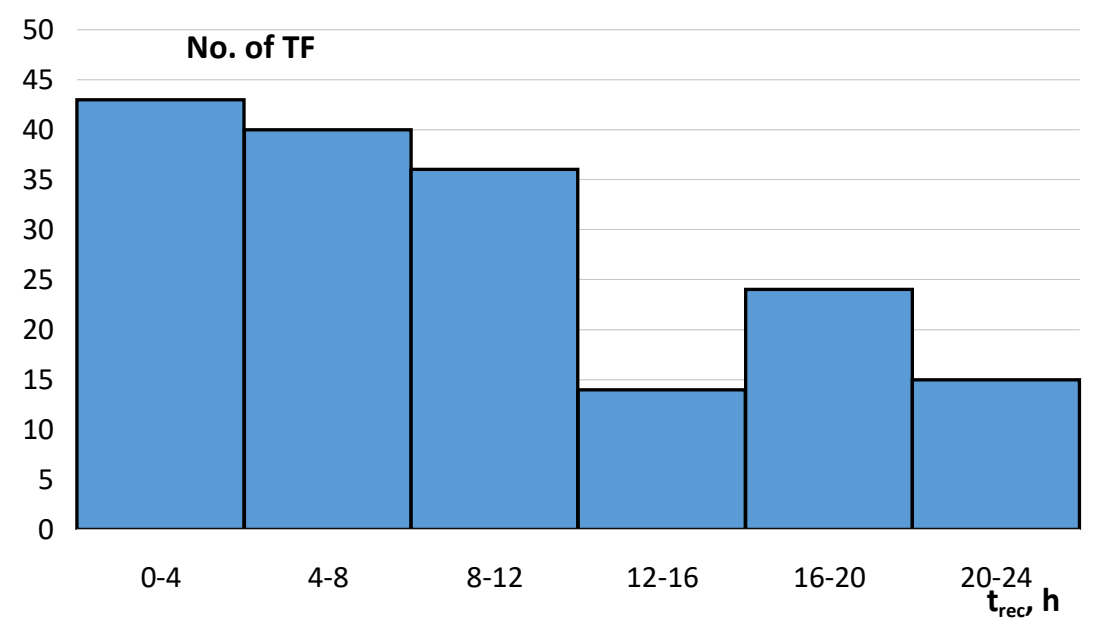

\section{Fig. 1. Distribution of the duration of the restoration of power supply} to consumers arising under unfavorable weather conditions

To obtain the distribution shown in Figure 1, the information on 10,000 failures in the enterprise Svetlogradskie Electric Networks of the Stavropol Territory for 2001-2011 has been analyzed. It should be noted that the main cause of failures in power supply systems is strong wind, accompanied either by thunderstorm with rainfall (in summer) or intense icing on wires (in winter).

The following equation has been used to analyze the effect on SAIDI of the recovery time of consumers' power supply:

$$
S A I D I=\frac{\sum T_{\text {STORMi }} N_{\text {STORMi }}+\sum T_{\text {COk }} N_{C O k}+\sum T_{S M n} N_{S M n}}{N_{\text {TOT }}},
$$

where $T_{\text {STORM }}$ - duration of interruptions in power supply to consumers in the amount of $N_{\text {STORM }}$, located in the $i$ node under the influence of adverse weather conditions;

$T_{C O k}$ - duration of interruptions in power supply to consumers in the amount of $N_{C O k}$, located in the $k$ node during current operation;

$T_{S M n}$ - duration of interruptions in power supply to consumers in the amount of $N_{S M n}$, located in the $n$ node when performing scheduled preventive work.

Thus, it is possible to estimate SAIDI using averaged values. Suppose that in the service area of distribution electric networks there are about $N_{T O T}=110000$ customers (100000 household consumers and 10000 enterprises and organizations), and one consumer means a connection point with authorized equipment for accounting for electric energy. Consumers are connected at low voltage $(0.4 \mathrm{kV})$. Considering that in Russia the radial scheme of distribution electric networks is mainly used, and the relay protection and automation and network management tools are concentrated mainly in power centers (at electrical substations), the minimum interruptible load volume in case of damage or scheduled work is the feeder and all consumer transformer substations connected to it. Therefore, the assumption that the average number of consumers on one feeder is a value $N_{C F}=200$. The average 
time to restore consumers' power supply during the current operation is $T_{C O}=4$ hours, the average time for scheduled maintenance is $T_{S M}=8$ hours, the average number of feeders at which scheduled preventive work is carried out during the year is $11 \%$ of the total, and those, in which failures occur under normal operating conditions, is $20 \%$ of the total.

The time to restore power supply in unfavorable weather conditions is of the utmost interest. Since, from the analysis of the information in Figure 1, it can be concluded that $25 \%$ of failures are eliminated in no more than 4 hours (the average recovery time is 2 hours), and the rest has an average recovery time of 12 hours. In this case, it is conditionally possible to divide all failures into two categories [6]: the first category of failures $(25 \%)$ can be eliminated by the forces of field service teams (FST), and the second category ( $75 \%$ ) require the use of complex repair and restoration work, and involvement not only FST, but also repair teams (RT). For the second part, the algorithm for restoring power supply is as follows - initially the damage is inspected by a FST ( $\bar{T}_{R E P 1}=2$ hours), then the work is performed by a RT ( $T_{R E P 2}=8$ hours), then the repaired equipment is turned on by FST $T_{T E P 3}=2$ hours.

It is possible to use the queueing theory to determine the number of FST and RT. [4]. For this, the following assumptions are applied. In general, the elimination of each interruption in power supply to consumers (hereinafter referred to as failure, and, as a result, the emergence of a requirement to eliminate the failure -a repair request) has its own technical features. In some cases, it is possible not to take these features into account, but to apply a queuing system (QS) [7], which allows for averaged parameters to determine the delay time for servicing repair requests depending on the number of personnel, which affects the reliability index of the power supply system SAIDI.

There are various types of QS [7], in our case we use one of the most common QS types $\left(M / M / N_{B R I G}\right):(G D / \infty / \infty)$ - the input flow is of Poisson type, distribution of the recovery time has an exponential distribution(with some assumptions, it is possible to calculate with any law of distribution of the recovery time), the number of teams is limited, there is a general queue, the queue size is not limited, an infinite number of requests can be generated for QS. The use of this type of QS is also possible with a sufficiently large number of failures that occur in networks in unfavorable weather conditions.

The initial data for the QS are: the failure flow parameter $\lambda$ (the flow of incoming requests to the QS); the average recovery time $T_{R E P}$ or the recovery intensity $\mu=1 / T_{R E P}$ (which is true for the applied QS according to [7]); the number of RB or FST $N_{B R I G}$. For calculations using the QS, a numerical solution of the system of differential equations describing the QS used has been performed. The elimination of interruptions in the power supply of consumers for different categories of failures is different: for the first category - restoration by the forces of FST, and for the second category, restoration takes place in several stages. When considering the elimination of failures, a multiphase QS model has been used (Figure 2), which consists of three phases: the first phase is the maintenance of failures by field service teams (FST), the second is the repair work by the repair teams (RT) and the third is the acceptance of the work performed by RT and performing of operational switching by the forces of FST. Accordingly, each of the phases is characterized by its own parameters: $\lambda, T_{R E P}, N_{B R I G}$. Passing the repair request for the second and third phase (separation of failures of the first and second category) is also a random process.

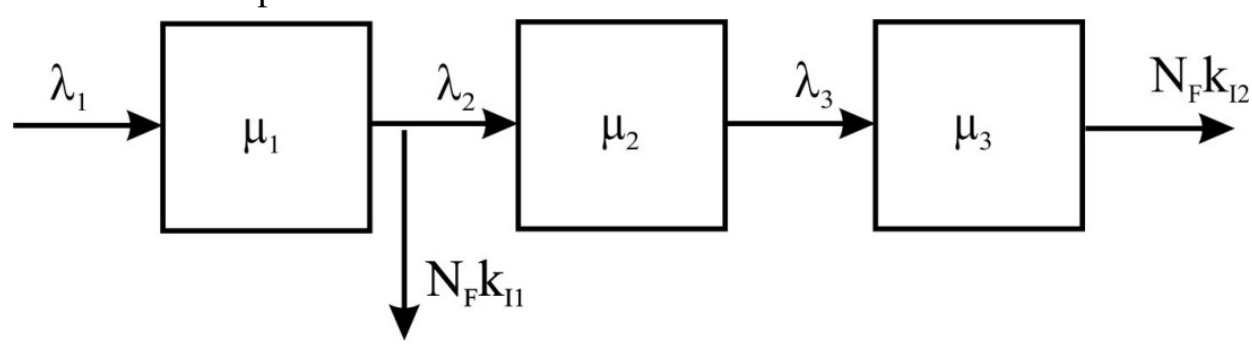

Fig. 2. Multiphase QS circuit

The numerical solution of the system of equations describing the QS has been carried out using the explicit $4^{\text {th }}$-order Runge-Kutta method. Moreover, the system of equations has a limited queue size $N_{Q}$, but it was shown in [8] that under the condition $4 \cdot N_{B R I G}<N_{Q}$ the finite value of the 
queue does not significantly affect the results of numerical solutions of the system of equations. The result of a numerical solution of the system of differential equations is an array of probabilities of all states of the system at each moment in time, based on these results, such a parameter as the time to wait for a service request $T_{Q}$ has been calculated. To calculate this parameter, the Little formula has been used [7]:

$$
T_{Q}(t)=\frac{1}{\lambda} L_{Q}(t)
$$

where $\lambda$-failure flow parameter;

$L_{\mathrm{Q}}-$ average number of requests in the queue, which can be determined by the formula:

$$
L_{Q}(t)=\sum_{k=1}^{N_{Q}} k P_{n_{B R / G}+k}(t) \text {. }
$$

To assess the effect of the teams' number $N_{\mathrm{BRIG}}$, involved in eliminating network failures on the reliability index SAIDI, the algorithm has been developed and the software has been implemented that allow the system of differential equations describing the QS to be compiled and solved numerically and processed the calculation results to obtain parameters, such as $T_{Q}(t)$ and $L_{Q}(t)$. The parameter $T_{Q}(t)$ allows us to calculate SAIDI reliability index by averaged values using formula (2), which takes the form:

$$
S A I D I=\frac{\bar{T}_{\text {STOT1 }} N_{F} \bar{N}_{C F} k_{I 1}+\bar{T}_{\text {STOT } 2} N_{F} \bar{N}_{C F} k_{I 2}+\bar{T}_{C O} \bar{N}_{C O}+\bar{T}_{S M} \bar{N}_{S M}}{N_{\text {TOT }}},
$$

where $T_{\text {STOT1 }}-$ first category average failure recovery time:

$$
\bar{T}_{\text {STOT } 1}=\bar{T}_{\text {S1stage }}=\bar{T}_{R E P 1}+\bar{T}_{Q 1 \text { stage }},
$$

$T_{\text {STOT2 }}$ - second category average failure recovery time:

$$
\bar{T}_{\text {STOT } 2}=\bar{T}_{\text {S1stage }}+\bar{T}_{\text {S2stage }}+\bar{T}_{\text {S3stage }},
$$

at

$$
\bar{T}_{S 2 \text { stage }}=\bar{T}_{R E P 2}+\bar{T}_{Q 2 \text { stage }}, \bar{T}_{S 3 \text { stage }}=\bar{T}_{R E P 3}+\bar{T}_{Q 3 \text { stage }},
$$

where $N_{F}$ - number of feeders disconnected due to unfavorable weather conditions;

$N_{C O}$ and $N_{S M}$ - average number of consumers for the year subjected to cutoffs due to failures during the current operation and scheduled maintenance work, respectively; $k_{11}$ and $k_{\mathrm{I} 2}$ - coefficients taking into account the distribution of network failures under the influence of unfavorable weather conditions in categories 1 and 2, respectively.

\section{Results and discussion}

The QS calculations have been performed on the basis of the averaged data obtained from the processing of information on the operation of electric networks. When calculating the QS, the phase parameters have been taken: $\lambda_{1}=1 \mathrm{~h}^{-1}, T_{R E P 1}=2$ hours, $\lambda_{2}=0.75 \mathrm{~h}^{-1}, T_{R E P 2}=8$ hours, $\lambda_{3}=0.75 \mathrm{~h}^{-1}$, $T_{R E P 3}=3$ hours. In addition, the total review period is 24 hours, the step size is 0.1 hour, the number of equations is $N_{E Q}=41$, the number of teams $N_{B R I G}$ varied from 1 to 8 , and the number of places in the queue ranged from 39 to 32 . The values are $k_{\mathrm{I} 1}=0.25$ and $k_{\mathrm{I} 2}=0.75$, the total number of failed feeders is taken to be $N_{F}=24$ (taking into account the failure rate and the duration of unfavorable weather conditions 24 hours). $N_{C O}=22000$ and $N_{S M}=12100$. The calculation results are presented in Table 1. Calculation of the value of SAIDIprovided that a very large number of teams are involved $\left(T_{Q 1 \text { stage }}, T_{Q 2 \text { stage }}, T_{Q 3 s t a g e}(t)=0\right.$ ) gives a value of about 2.1 hours, and without taking into account failures caused by unfavorable weather conditions 1.68 hours.

The data in Table 1have been obtained as follows. The second, third, and fourth columnsare the waiting time for the service request $-T_{Q}(t)$, taken at time $t=24$ hours after the start of the period of failures with a given intensity $\lambda_{1}$, when unfavorable weather conditions occurred. This time has been determined for each of the phases of the QS. From the fifth to seventh columns, the values have been obtained using expressions (6) and (8) and show the average time of requests passing through the QS 
phases, and the parameters in the eighth and ninth columns have been calculated from expressions (6) and (7) and show the total average failure elimination time of the first and second categories, respectively. As it can be seen from Table 1, an increase in the number of teams leads to a decrease in the value of SAIDI and an improvement in the reliability of power supply to consumers.

Table 1

Calculation results for SAIDI based on averaged values (in hours)

\begin{tabular}{|c|c|c|c|c|c|c|c|c|c|}
\hline $\boldsymbol{N}_{\text {BRIG }}$ & $\boldsymbol{T}_{\boldsymbol{Q} \text { 1stage }}$ & $\boldsymbol{T}_{\mathbf{Q}_{\text {stage }}}$ & $\boldsymbol{T}_{\text {Qstage }}$ & $\boldsymbol{T}_{\boldsymbol{S 1 s t a g e}}$ & $\boldsymbol{T}_{\text {S2stage }}$ & $\boldsymbol{T}_{\boldsymbol{S 3 s t a g e}}$ & $\boldsymbol{T}_{\text {STOT1 }}$ & $\boldsymbol{T}_{\text {STOT2 }}$ & SAIDI \\
\hline 1 & 11.99 & 18.93 & 8.98 & 13.99 & 26.93 & 10.98 & 13.99 & 51.90 & 3.53 \\
\hline 2 & 3.69 & 14.22 & 1.80 & 5.69 & 22.22 & 3.80 & 5.69 & 31.71 & 2.78 \\
\hline 3 & 0.80 & 10.53 & 0.31 & 2.80 & 18.53 & 2.31 & 2.80 & 23.63 & 2.48 \\
\hline 4 & 0.17 & 6.56 & 0.06 & 2.17 & 14.56 & 2.06 & 2.17 & 18.79 & 2.32 \\
\hline 5 & 0.04 & 3.96 & 0.0115 & 2.04 & 11.96 & 2.01 & 2.04 & 16.01 & 2.23 \\
\hline 6 & 0.01 & 2.21 & 0.0021 & 2.01 & 10.21 & 2.00 & 2.01 & 14.22 & 2.17 \\
\hline 7 & 0.0019 & 1.15 & 0.0004 & 2.00 & 9.15 & 2.00 & 2.00 & 13.15 & 2.13 \\
\hline 8 & 0.0004 & 0.56 & 0.0001 & 2.00 & 8.56 & 2.00 & 2.00 & 12.56 & 2.11 \\
\hline
\end{tabular}

\section{Conclusions}

The paper assesses the impact of the number of personnel involved in the elimination of mass failures in electrical networks that occur when exposed to unfavorable weather conditions. On the example of one network enterprise, the average indexes have been assessed by the number and duration of disconnection of consumers during current operation and the performance of scheduled preventive work. The evaluation of SAIDI, when using average operation data and the calculation results of parameters, such as the waiting time for service requests for repairs, using the queuing system, has shown that an increase in the number of teams can significantly increase this index. Thus, an increase in the number of teams eliminating interruptions from three ( 2 field service teams and 1 repair team) to twenty ( 8 field service teams and 4 repair teams) reduces SAIDI, calculated over the year of operation from 3. 53 hours to 2.32 hours.

\section{References}

[1] Khalid M., Akram U. Email Author, Shafiq, S. Optimal planning of multiple distributed generating units and storage in active distribution networks. IEEE Access, vol. 6, 2018, pp. 55234-55244.

[2] Lu Z., Li D., Lü X., Zhao H., Hou X. Multiple faults repair strategy under ice storm for distribution network with distributed generators. Diangong Jishu Xuebao, vol. 33(2), 2018, pp. 423-432.

[3] IEEE Std 1366"TM-2003(Revision of IEEE Std 1366-1998) IEEE Guide for Electric Power Distribution Reliability Indices Transmission and Distribution Committee of the IEEE Power Engineering Society Published by The Institute of Electrical and Electronics Engineers, Inc., USA

[4] Billinton R., AllanR. N. Reliability evaluation of power systems second edition. Plenum press. New York and London, 1996. 534 p.

[5] Hadjsaid N. Electrical Distribution Networks. Wiley-IEEE Press, 2011. 512 p.

[6] A. V. Efanov, S. V. Oskin, S. S. Yastrebov, V. G. Zhdanov, V. N. Shemyakin. Determining the number of staff to eliminate the results of emergency situations of natural and anthropogenic origin in rural electrical networks. RJPBCS, vol. 9(4), 2018, pp. 559-564.

[7] Taha H.A. Operations research: an introduction. University of Arkansas, Fayetteville: ( Pearson Education Limited, 2017 Paerson, 2017. 849 p.

[8] Ефанов А. В., Оськин С. В., Ястребов С. С, Ярош В. А., Букреев А. Г. Сравнение стационарных и численных решений систем массового обслуживания при решении задач ликвидации последствий чрезвычайных ситуаций в сельских электрических сетях (Comparison of stationary and numerical solutions of Queuing systems for solving problems of emergency response in rural electric networks ). Selskiy Mechanizator. 2019. No. 4. pp. 22-24. (In Russian). 